\title{
Slip Effects on Pulsatile Flow of Blood through a Stenosed Arterial Segment under Periodic Body Acceleration
}

\author{
A. Sinha, G. C. Shit, and P. K. Kundu \\ Department of Mathematics, Jadavpur University, Kolkata 700032, India \\ Correspondence should be addressed to G. C. Shit; gopal_iitkgp@yahoo.co.in
}

Received 17 June 2013; Accepted 7 July 2013

Academic Editors: A. Cappozzo and D. S. Naidu

Copyright (C) 2013 A. Sinha et al. This is an open access article distributed under the Creative Commons Attribution License, which permits unrestricted use, distribution, and reproduction in any medium, provided the original work is properly cited.

\begin{abstract}
A theoretical investigation concerning the influence of externally imposed periodic body acceleration on the flow of blood through a time-dependent stenosed arterial segment by taking into account the slip velocity at the wall of the artery has been carried out. A mathematical model is developed by treating blood as a non-Newtonian fluid obeying the Casson fluid model. The pulsatile flow is analyzed by considering a periodic pressure gradient and the inertial effects as negligibly small. A suitable generalized geometry for time-dependent stenosis is taken into account. Perturbation method is used to solve the coupled implicit system of nonlinear differential equations that govern the flow of blood. Analytical expressions for the velocity profile, volumetric flow rate, and wall shear stress are obtained. A thorough quantitative analysis has been made through numerical computations of the variables involved in the analysis that are of special interest in this study. The computational results are presented graphically. The results for different values of the parameters involved in the problem under consideration presented here show that the flow is appreciably influenced by slip velocity in the presence of periodic body acceleration.
\end{abstract}

\section{Introduction}

There are number of evidences available in the scientific literatures that vascular fluid dynamics plays a major role in the development and progression of arterial diseases. Local narrowing in the lumen of an arterial segment is commonly referred to as stenosis. This occurs due to deposition of various substances like cholesterol on the endothelium of arterial wall. When an obstruction is developed in an artery, one of the most serious consequences is the increased resistance and the associated reduction of the blood flow to the particular vascular bed supplied by the artery.

Thus, the presence of a stenosis leads to stroke, heart attack, and serious circulatory disorders. Different studies on the flow of blood through arterial segments with obstruction have been carried out experimentally and theoretically by several investigators [1-7]. The assumption of Newtonian behavior of blood is acceptable for high shear rate flow through larger arteries [4]. But, blood, being a suspension of cells in plasma, exhibits non-Newtonian behavior at low shear rate $(\dot{\gamma}<10 / s)$ in small diameter arteries $(0.02-$ $0.1 \mathrm{~mm}$ ) [8]. Several studies were performed to analyze the steady flow of blood, treating it as a Newtonian fluid $[9,10]$. It is well known that blood flow in the human circulatory system is caused by the pumping action of the heart, which in turn produces a pulsatile pressure gradient throughout the system [11]. Human heart is a muscular pump and due to contraction and expansion of heart muscles, there produces a pressure difference in its systolic and diastolic conditions, popularly known as pressure pulse which physicians check at the wrist. The cyclic nature of heart pump creates pulsatile conditions in all arteries. The ejects and fills with blood in alternating cycles are called systolic and diastolic. Blood is pumped out of the heart during systolic, whereas the heart rests during diastole and no blood is ejected. Pressure and flow rate are characteristic in pulsatile shapes that vary in different parts of the arterial system. Thus, several researchers have studied pulsatile flow of blood, treating it as a Newtonian fluid [12-14]. Long et al. [8] numerically investigated the pulsatile flow behaviour of blood in the poststenotic region by considering inlet diameter as $8 \mathrm{~mm}$, prestenotic length $48 \mathrm{~mm}$, poststenosis domain $180 \mathrm{~mm}$, and stenosis length $16.07 \mathrm{~mm}$. Clark [1] performed the experimental studies of the pulsatile flow in a model of aortic stenosis taking the 
Reynolds number 740 and 2000. Nagarani and Sarojamma [15] developed a mathematical model of pulsatile flow of Casson fluid for blood flow through stenosed narrow arteries. They used perturbation technique to solve their problem. Siddiqui et al. [16] mathematically analyzed the flow of blood through narrow arteries by considering Herschel-Bulkley fluid model as well as Casson fluid model.

In our daily life, we often face some external body acceleration, such as traveling in high velocity vehicles and aircrafts. In various sports during the performance, a high acceleration/vibration suddenly takes place. These types of situations undoubtedly affect the normal flow of blood which lead to headache, vomiting tendency, loss of vision, abnormality in pulse rate, and so forth. Therefore, it is necessary to maintain such type of body accelerations to avoid these types of health hazards. Due to physiological importance of body acceleration, many theoretical investigations are developed for the flow of blood under the influence of body acceleration with and with out stenosis. Sud and Sekhon [17] made an analysis on blood flow under the time-dependent acceleration. They pointed out that the high blood velocity and high shear rate are capable of harming the circulation which is produced under the influence of such time-dependent acceleration. Sud and Sekhon [18] also analyzed the blood flow through a model of the human arterial system under the influence of periodic acceleration. They observed that the body acceleration has an enhancing effect on the flow rate. Chaturani and Palanisamy [19] investigated the pulsatile flow of blood under the influence of periodic body acceleration by treating blood as a Power-law fluid. Majhi and Nair [20] studied the pulsatile flow of blood under the influence of body acceleration by assuming blood as a third grade fluid. Shit and Roy [21] examined the effect of externally imposed body acceleration and magnetic field on pulsatile flow of blood through an arterial segment having stenosis with noslip velocity condition.

Misra et al. [22] conducted a theoretical study concerning blood flow through a stenosed arterial segment wherein they considered no-slip condition at the vessel wall. There are, however, numerous situations where there may be a partial slip between the fluid and the boundary. For many fluids, the motion of the particulate fluid is still governed by the NavierStokes equations, but the usual no-slip condition at the boundary should be replaced by the slip condition [2]. Misra and Shit [23] carried out the role of slip velocity in blood flow through stenosed arteries. Several authors [24, 25] suggested the presence of a red blood cell occurring in slip condition at the vessel wall. Recently, Ponalagusamy [26] and Biswas and Chakraborty [27, 28] have developed mathematical models for blood flow through stenosed arterial segment, by taking a velocity slip condition at the constricted wall. Thus, it seems that consideration of a velocity slip at the stenosed vessel wall will be quite rational in blood flow modeling. However, the effect of body acceleration on pulsatile flow of blood through an arterial segment having time-dependent stenosis in the presence velocity-slip has not been considered so far to the best of our knowledge.

The motivation of this paper is to study the unsteady flow of blood through an arterial segment with time-dependent

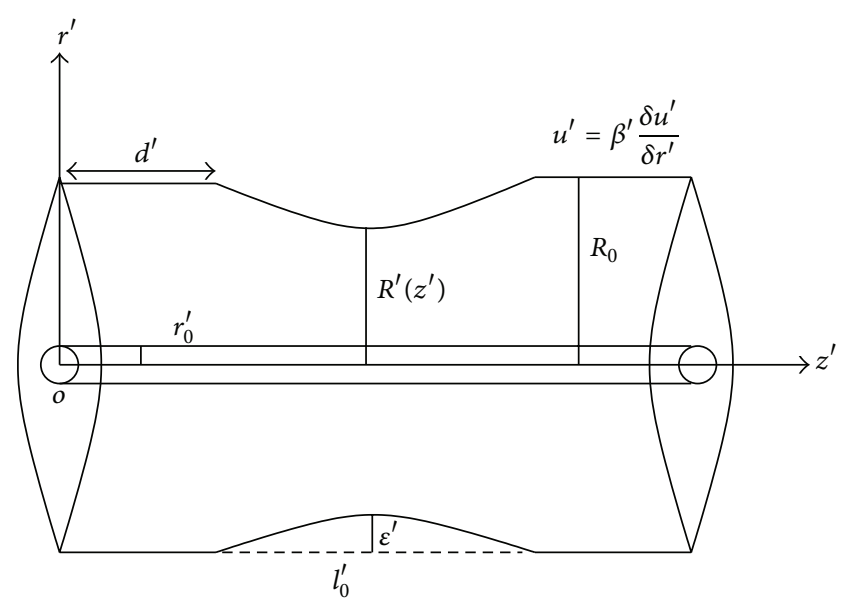

FIGURE 1: Schematic diagram of the model geometry.

stenosis in the presence of velocity slip. The analysis is carried out by employing appropriate analytical methods and some important predictions have been made on the basis of the present study. In order to illustrate the applicability of the theoretical analysis, the derived analytical expressions have been computed for a specific situation, with an aim to observe the variation of some quantities of special interest. The computed values are reported graphically.

\section{Formulation of the Problem}

Let us consider an axially symmetric incompressible laminar pulsatile and fully developed flow of blood through an arterial segment with time-dependent stenosis as shown in Figure 1. Although the modelling of blood flow in arteries may appear to be more intuitive by considering tube flow, it is worthwhile mentioning that the flow in a tube resembles the flow behavior in a channel in many situations $[29,30]$. With this rationale, the present problem is formulated by considering flow through a channel. Body acceleration and slip effect are taken into account for the present problem. Blood flowing in arteries is considered here as a suspension of erythrocytes (red blood cells) in plasma. It is assumed that the fluid is uniformly dense throughout. Here blood is represented by Casson fluid model. The length of the artery is assumed to be large enough as compared to its radius so that at the entrance and exit sections, special wall effects can be neglected. It has been reported that the radial velocity is negligibly small for a low Reynolds number flow in a narrow artery with stenosis [31].

With the above considerations, the equations that govern the flow of blood may be put in the form

$$
\begin{gathered}
\rho \frac{\partial u^{\prime}}{\partial t^{\prime}}=-\frac{\partial p^{\prime}}{\partial z^{\prime}}-\frac{1}{r^{\prime}} \frac{\partial}{\partial r^{\prime}}\left(r^{\prime} \tau^{\prime}\right)+G^{\prime}\left(t^{\prime}\right), \\
0=\frac{\partial p^{\prime}}{\partial r^{\prime}},
\end{gathered}
$$

where $u^{\prime}$ is the axial component of blood velocity, $p^{\prime}$ is the pressure, $\rho$ is the density of blood, $\tau^{\prime}$ is the shear stress, and 
$G^{\prime}\left(t^{\prime}\right)$ is the body acceleration. The constitutive equation of Casson fluid (which represents the blood) is given by

$$
\begin{gathered}
-\frac{\partial u^{\prime}}{\partial r^{\prime}}=\frac{1}{\mu}\left(\tau^{1 / 2}-\tau_{y}^{1 / 2}\right)^{2}, \quad r_{0}^{\prime} \leq r^{\prime} \leq R^{\prime}, \\
\frac{\partial u^{\prime}}{\partial r^{\prime}}=0, \quad 0 \leq r^{\prime} \leq r_{0}^{\prime},
\end{gathered}
$$

where $\tau_{y}$ is the yield stress and $\mu$ is the coefficient of viscosity.

Let us consider a generalized geometry of time-dependent single stenosis (cf. Figure 1) as

$$
\begin{aligned}
& R^{\prime}\left(z^{\prime}, t^{\prime}\right)=R_{0}\left[1-\frac{\epsilon^{\prime}\left(1-e^{-t^{\prime} / T^{\prime}}\right)}{R_{0} l_{0}^{\prime n}} \frac{n^{n /(n-1)}}{n-1}\right. \\
& \left.\times\left(l_{0}^{\prime n-1}\left(z^{\prime}-d^{\prime}\right)-\left(z^{\prime}-d^{\prime}\right)^{n}\right)\right], \\
& d^{\prime} \leq z^{\prime} \leq l_{0}^{\prime}+d^{\prime}
\end{aligned}
$$

in which $R^{\prime}\left(z^{\prime}, t^{\prime}\right)$ is the radius of the arterial segment in the stenotic region at an axial distance $z^{\prime}$ at a time $t^{\prime}, R_{0}$ is the radius of a normal portion of the artery, $l_{0}^{\prime}$ is the length of the stenosis, $d^{\prime}$ indicates the location, $n$ is a parameter that determines the shape of the stenosis, and $\epsilon^{\prime}$ is the maximum height of the stenosis located at

$$
z^{\prime}=d^{\prime}+\frac{l_{0}^{\prime}}{\left(n^{1 /(n-1)}\right)} .
$$

The ratio of the height of the stenosis to the radius of the normal portion of the artery is considered to be much less than unity.

The boundary conditions for the present problem may be put mathematically in the form

$$
\begin{gathered}
u^{\prime}=\beta^{\prime} \frac{\partial u^{\prime}}{\partial r^{\prime}} \text { at } r^{\prime}=R^{\prime}\left(z^{\prime}\right), \\
\tau^{\prime} \text { is finite at } r^{\prime}=0,
\end{gathered}
$$

where $\beta^{\prime}$ denotes the slip length. by

The periodic body acceleration in axial direction is given

$$
G^{\prime}\left(t^{\prime}\right)=a_{0} \cos \left(\omega_{1}^{\prime} t^{\prime}+\phi\right)
$$

where $a_{0}$ is the amplitude, $\omega_{1}^{\prime}=2 \pi f_{1}^{\prime}, f_{1}^{\prime}$ is the frequency in $\mathrm{Hz}$, is assumed to be small so that the wave effect can be neglected, and $\phi$ is the lead angle of $G^{\prime}\left(t^{\prime}\right)$ with respect to the heart action.

Since the pressure gradient is a function of $z^{\prime}$ and $t^{\prime}$, we can take

$$
-\frac{\partial p^{\prime}\left(z^{\prime}, t^{\prime}\right)}{\partial z^{\prime}}=A_{0}+A_{1} \cos \left(\omega_{2}^{\prime} t^{\prime}\right),
$$

where $A_{0}$ and $A_{1}$, respectively, are the steady component and the amplitude of the fluctuating component of the pressure gradient and $\omega_{2}^{\prime}=2 \pi f_{2}^{\prime}, f_{2}^{\prime}$ is the pulse frequency in $\mathrm{Hz}$.

Let us introduce the following nondimensional variables:

$$
\begin{gathered}
z=\frac{z^{\prime}}{R_{0}}, \quad R=\frac{R^{\prime}}{R_{0}}, \quad r=\frac{r^{\prime}}{R_{0}}, \quad d=\frac{d^{\prime}}{R_{0}}, \\
l_{0}=\frac{l_{0}^{\prime}}{R_{0}}, \quad r_{0}=\frac{r_{0}^{\prime}}{R_{0}}, \quad \epsilon=\frac{\epsilon^{\prime}}{R_{0}}, \\
\tau=\frac{2 \tau^{\prime}}{A_{0} R_{0}}, \quad \theta=\frac{2 \tau_{y}}{A_{0} R_{0}}, \quad \omega=\frac{\omega_{1}^{\prime}}{\omega_{2}^{\prime}}, \\
t=\omega_{2}^{\prime} t^{\prime}, \quad T=\omega_{2}^{\prime} T^{\prime}, \quad B=\frac{a_{0}}{A_{0}}, \quad \alpha^{2}=\frac{R_{0}^{2} \omega_{2}^{\prime} \rho}{\mu}, \quad \beta=\frac{\beta^{\prime}}{A_{0}} .
\end{gathered}
$$

Using the nondimensional variables defined in (10), (1), (3), and (4), respectively, reduces to

$$
\begin{gathered}
\alpha^{2} \frac{\partial u}{\partial t}=4(1+e \cos t)+4 B \cos (\omega t+\phi)-\frac{2}{r} \frac{\partial(r \tau)}{\partial r} \\
\frac{\partial u}{\partial r}=0 \quad 0 \leq r \leq r_{0} \\
\tau^{1 / 2}=\theta^{1 / 2}+\frac{1}{\sqrt{2}}\left(-\frac{\partial u}{\partial r}\right)^{1 / 2} \quad r_{0} \leq r \leq R .
\end{gathered}
$$

Similarly, the boundary conditions (7) are also transformed to

$$
u=\beta \frac{\partial u}{\partial r} \quad \text { at } r=R(z),
$$

$\tau$ is finite at $r=0$,

where

$$
\begin{aligned}
R(z, t)=1 & -\frac{\epsilon\left(1-e^{-t / T}\right)}{l_{0}^{n}} \frac{n^{n /(n-1)}}{n-1} \\
& \times\left(l_{0}^{n-1}(z-d)-(z-d)^{n}\right), \quad d \leq z \leq l_{0}+d .
\end{aligned}
$$

\section{Analytical Solution}

Considering the Womersley parameter to be small $\left(\alpha^{2} \ll 1\right)$, the axial velocity component $u$, shear stress $\tau$, plug core radius $r_{0}$, and plug core velocity $u_{p}$ are expressed in the following form:

$$
\begin{gathered}
u=u_{0}+\alpha^{2} u_{1}+\cdots, \\
\tau=\tau_{0}+\alpha^{2} \tau_{1}+\cdots, \\
r_{0}=r_{00}+\alpha^{2} r_{10}+\cdots, \\
u_{p}=u_{0 p}+\alpha^{2} u_{1 p}+\cdots,
\end{gathered}
$$


Using (17) and (18) in (11), we get

$$
\begin{gathered}
\frac{\partial\left(r \tau_{0}\right)}{\partial r}=2 r g(t), \\
\frac{\partial u_{0}}{\partial t}=-\frac{2}{r} \frac{\partial\left(r \tau_{1}\right)}{\partial r},
\end{gathered}
$$

where $g(t)=(1+e \cos t)+B \cos (\omega t+\phi)$.

Integrating (21) and using the boundary condition (15), we obtain

$$
\tau_{0}=r^{2} g(t)
$$

Using (17) and (18), (12) can be written as

$$
\begin{gathered}
-\frac{\partial u_{0}}{\partial r}=2\left(\theta+\tau_{0}-2 \sqrt{\theta \tau_{0}}\right), \\
-\frac{\partial u_{1}}{\partial r}=2 \tau_{1}\left(1-\sqrt{\frac{\theta}{\tau_{0}}}\right) .
\end{gathered}
$$

Substituting (17) in (14), we obtain

$$
\begin{aligned}
& u_{0}=\beta \frac{\partial u_{0}}{\partial r} \quad \text { at } r=R, \\
& u_{1}=\beta \frac{\partial u_{1}}{\partial r} \quad \text { at } r=R .
\end{aligned}
$$

Using (23) and the boundary condition (26), the solution of (24) yields,

$$
\begin{aligned}
u_{0}= & g\left(R^{2}-r^{2}\right)-\frac{8 \sqrt{\theta g}}{3}\left(R^{3 / 2}-r^{3 / 2}\right) \\
& +2 \theta(R-r)-2 \beta g R+4 \sqrt{R g \theta}-2 \beta \theta .
\end{aligned}
$$

Plug velocity can be obtained from (28) by putting $r=r_{00}$ as

$$
\begin{aligned}
u_{0 p}= & g\left(R^{2}-r_{00}^{2}\right)-\frac{8 \sqrt{\theta g}}{3}\left(R^{3 / 2}-r_{00}^{3 / 2}\right) \\
& +2 \theta\left(R-r_{00}\right)-2 \beta g R+4 \sqrt{R g \theta}-2 \beta \theta .
\end{aligned}
$$

Using the relation (28) and boundary condition (15) in (22), we obtain the expression for $\tau_{1}$ as

$$
\begin{aligned}
\tau_{1}=\frac{g^{\prime} R^{3}}{8} & {\left[2\left(\frac{r}{R}\right)-\left(\frac{r}{R}\right)^{3}-\frac{3}{8} \sqrt{\frac{\theta}{g R}}\right.} \\
& \left.\times\left(7\left(\frac{r}{R}\right)-4\left(\frac{r}{R}\right)^{5 / 2}\right)\right] \\
- & \frac{g^{\prime} \beta R}{2}\left(1-\sqrt{\frac{\theta}{g R}}\right) r .
\end{aligned}
$$

Using (23) and (30) and the boundary condition (27), from (25), we obtain the expression for $u_{1}$ as

$$
\begin{aligned}
& u_{1}=\frac{g^{\prime} R^{4}}{8}\left[\left(\frac{r}{R}\right)^{4}-4\left(\frac{r}{R}\right)^{2}+3+\sqrt{\frac{\theta}{g R}}\right. \\
& \times\left(\frac{16}{3}\left(\frac{r}{R}\right)^{2}-\frac{424}{127}\left(\frac{r}{R}\right)^{7 / 2}\right. \\
& \left.+\frac{16}{3}\left(\frac{r}{R}\right)^{3 / 2}-\frac{1144}{147}\right) \\
& \left.+\frac{\theta}{g R}\left(\frac{128}{63}\left(\frac{r}{R}\right)^{3}-\frac{64}{9}\left(\frac{r}{R}\right)^{3 / 2}+\frac{320}{63}\right)\right] \\
& +\frac{g^{\prime} R \beta}{2}\left[2 R\left(\sqrt{\frac{\theta}{g R}}-1\right)\right. \\
& \times\left(\left(1-\frac{8}{7} \sqrt{\frac{\theta}{g R}}\right) R-\beta\left(1-\sqrt{\frac{\theta}{g R}}\right)\right) \\
& -\left(1-\sqrt{\frac{\theta}{g R}}\right)\left(R^{2}-r^{2}\right)+\frac{4}{3} \sqrt{\frac{\theta}{g}} \\
& \left.\times\left(1-\sqrt{\frac{\theta}{g R}}\right)\left(R^{3 / 2}-r^{3 / 2}\right)\right] .
\end{aligned}
$$

The expression for $u_{1 p}$ can be obtained from (31) when $r=r_{10}$ as

$$
\begin{aligned}
& u_{1 p}=\frac{g^{\prime} R^{4}}{8}\left[\left(\frac{r_{10}}{R}\right)^{4}-4\left(\frac{r_{10}}{R}\right)^{2}+3+\sqrt{\frac{\theta}{g R}}\right. \\
& \times\left(\frac{16}{3}\left(\frac{r_{10}}{R}\right)^{2}-\frac{424}{127}\left(\frac{r_{10}}{R}\right)^{7 / 2}\right. \\
& \left.+\frac{16}{3}\left(\frac{r_{10}}{R}\right)^{3 / 2}-\frac{1144}{147}\right)+\frac{\theta}{g R} \\
& \left.\times\left(\frac{128}{63}\left(\frac{r_{10}}{R}\right)^{3}-\frac{64}{9}\left(\frac{r_{10}}{R}\right)^{3 / 2}+\frac{320}{63}\right)\right] \\
& +\frac{g^{\prime} R \beta}{2}\left[2 R\left(\sqrt{\frac{\theta}{g R}}-1\right)\right. \\
& \times\left(\left(1-\frac{8}{7} \sqrt{\frac{\theta}{g R}}\right) R-\beta\left(1-\sqrt{\frac{\theta}{g R}}\right)\right) \\
& -\left(1-\sqrt{\frac{\theta}{g R}}\right)\left(R^{2}-r_{10}^{2}\right)+\frac{4}{3} \sqrt{\frac{\theta}{g}} \\
& \left.\times\left(1-\sqrt{\frac{\theta}{g R}}\right)\left(R^{3 / 2}-r_{10}^{3 / 2}\right)\right] .
\end{aligned}
$$


The total velocity distribution can be written as

$$
\begin{aligned}
& u=g\left(R^{2}-r^{2}\right)-\frac{8 \sqrt{\theta g}}{3}\left(R^{3 / 2}-r^{3 / 2}\right) \\
& +2 \theta(R-r)-2 \beta g R+4 \sqrt{R g \theta}-2 \beta \theta+\frac{\alpha^{2} g^{\prime} R^{4}}{8} \\
& \times\left[\left(\frac{r}{R}\right)^{4}-4\left(\frac{r}{R}\right)^{2}+3+\sqrt{\frac{\theta}{g R}}\right. \\
& \times\left(\frac{16}{3}\left(\frac{r}{R}\right)^{2}-\frac{424}{127}\left(\frac{r}{R}\right)^{7 / 2}\right. \\
& \left.+\frac{16}{3}\left(\frac{r}{R}\right)^{3 / 2}-\frac{1144}{147}\right) \\
& \left.+\frac{\theta}{g R}\left(\frac{128}{63}\left(\frac{r}{R}\right)^{3}-\frac{64}{9}\left(\frac{r}{R}\right)^{3 / 2}+\frac{320}{63}\right)\right] \\
& +\frac{g^{\prime} R \beta}{2}\left[2 R\left(\sqrt{\frac{\theta}{g R}}-1\right)\right. \\
& \times\left(\left(1-\frac{8}{7} \sqrt{\frac{\theta}{g R}}\right) R-\beta\left(1-\sqrt{\frac{\theta}{g R}}\right)\right) \\
& -\left(1-\sqrt{\frac{\theta}{g R}}\right)\left(R^{2}-r^{2}\right)+\frac{4}{3} \sqrt{\frac{\theta}{g}} \\
& \left.\times\left(1-\sqrt{\frac{\theta}{g R}}\right)\left(R^{3 / 2}-r^{3 / 2}\right)\right] .
\end{aligned}
$$

The nondimensional volumetric flow rate $Q$ is given by

$$
\begin{aligned}
Q(t)= & 4 \int_{0}^{R} u r d r=g R^{4}-\frac{16}{7} \sqrt{\frac{g \theta}{R}} R^{4} \\
+ & \frac{1}{3} R^{3} \theta+\frac{1}{12} R^{6} \alpha^{2} g^{\prime}-\frac{15}{77} \sqrt{\frac{\theta}{g R}} R^{6} \alpha^{2} g^{\prime} \\
+ & \frac{4 \theta R^{6} \alpha^{2} g^{\prime}}{35 g R}+g \beta R^{3}\left(1-\sqrt{\frac{\theta}{g R}}\right)^{2}+\beta g^{\prime} R^{5} \\
\times & {\left[\left(\sqrt{\frac{\theta}{g R}}-1\right)\right.} \\
& \times\left(\left(1-\frac{8}{7} \sqrt{\frac{\theta}{g R}}\right)-\frac{\beta}{R}\left(1-\sqrt{\frac{\theta}{g R}}\right)\right) \\
& \left.-\frac{1}{4}\left(1+\sqrt{\frac{\theta}{g R}}\right)+\frac{2}{7} \sqrt{\frac{\theta}{g R}}\left(1-\sqrt{\frac{\theta}{g R}}\right)\right]
\end{aligned}
$$

where $Q=Q^{\prime} /\left(\pi A_{0} R_{0}^{4} / 2 \mu\right), Q^{\prime}$ is the volumetric flow rate.
In dimensionless form, resistance to the flow is given by

$$
\lambda(t)=\frac{P_{0}-P_{L}}{Q}=\frac{L(1+e \cos (t+\phi))}{Q},
$$

where $P=P_{0}$ at $z=0$ and $P=P_{L}$ at $z=L$.

\section{Results and Discussion}

With a view to illustrate the applicability of the mathematical model developed and analyzed in the preceding sections, the analytical expressions for the axial velocity profile, wall shear stress, and volumetric flow rate are presented by taking into account the velocity-slip condition at the arterial wall. In order to get a proper insight into the flow behaviour of blood through a time-dependent stenosed arterial segment under body acceleration, the variations of $u, Q, \tau$, and $\lambda$ have been estimated and the computed results are presented in graphical form. The flowing blood is modeled as Casson fluid model. The governing equations of the flow are solved using perturbation analysis with the assumption that the Womersley frequency parameter is small which is valid for physiological situations in small blood vessels. In our analysis, the value of the shape parameter $(n)$ of the stenosis is taken to be 2 . The maximum height of the stenosis is generally taken as 0.3 and only to pronounce its effect, we have taken the range from 0.0 to 0.6 . The values of the nondimensional yield stress $\theta$ for the blood of the normal subject are between 0.01 and 0.03 and in diseased state, it is quite high and in such a case, the value of the yield stress is taken to lie between 0.1 and 0.4 . The velocity slip parameter is taken between -2.0 and -0.5 . The pressure gradient parameter $(e)$ is taken in the range $0.1-$ 0.4 . For this problem, the value of pulsatile Reynolds number is taken as 0.05 . To discuss the effects of the body acceleration parameter $B$ on the various flow quantities, its value is taken in the range between 0.0 and 0.8 .

Figures 2-6 give an idea of the axial velocity distribution in the case of blood flow in the vicinity of the stenotic portion of the arterial segment under the purview of the present study. The presence of velocity slip at the wall alters the blood velocity significantly as shown in Figure 2 . We observe from this figure that blood velocity decreases as the slip length increases. It is interesting to note that at $z=1.0$ (i.e., at the throat the stenosis) and at a particular time $t=\pi / 4$, for large values of slip length $(\beta=-1.0,-1.2)$ axial velocity increases with the radial distance, whereas the reverse trend is observed for $\beta=-0.5,-0.8$. Figure 3 represents the axial velocity distribution at a particular time $t=\pi / 4$ with the radial distance for different values of body acceleration parameter $B$. It is seen that at the throat of the stenosis (i.e., $z=1.0$ ), axial velocity monotonically decreases with the radial distance. From the same figure, we observed that the body acceleration parameter $B$ brings quantitative as well as qualitative changes in velocity profiles. It reveals that the velocity increases as the the body acceleration parameter increases. This result supports the phenomenon that body acceleration reduces the flow resistance and so the velocity of blood flow increases with the increase in body acceleration. Figure 4 gives the distribution of the axial velocity at the onset of the stenosis for 


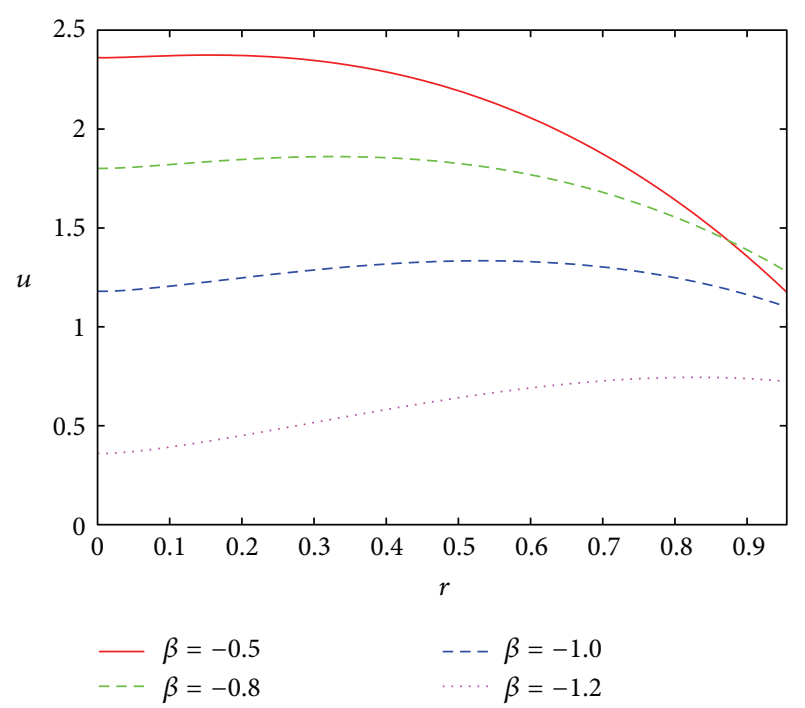

Figure 2: Velocity distribution for different values of $\beta$ when $n=$ $2, e=0.2, B=0.4, \alpha^{2}=0.05, \epsilon=0.3, \omega=2.0, \phi=0.0, \theta=$ 0.1 , and $t=\pi / 4$.

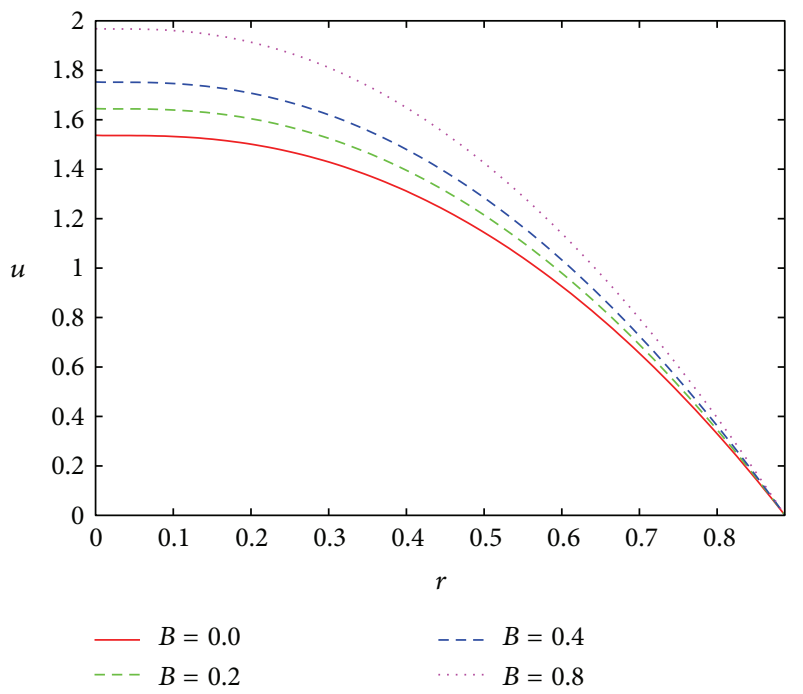

FIgURE 3: Velocity distribution for different values of $B$ when $n=2$, $e=0.2, \beta=0.0, \alpha^{2}=0.05, \epsilon=0.3, \omega=2.0, \phi=0.0, \theta=0.1$, and $t=\pi / 4$.

different values of yield stress. This figure depicts that velocity decreases with increasing the yield stress. It is also noted that velocity profile oscillates with time. Figure 5 depicts the distribution of the velocity at the throat of the stenosis with time for different values of the height of the stenosis. It has been observed from this figure that for any value of $\epsilon$, the maximum value attains on the axis of symmetry. Figure 6 illustrates the velocity distribution at $z=1.5$ for different values of the pressure gradient parameter $e$. This figure reveals that velocity increases as the pressure gradient parameter $e$ increases when $t \in[0.0,0.95],[4.1,7.4]$, and so on, whereas the reverse trend is observed in rest of the time intervals.

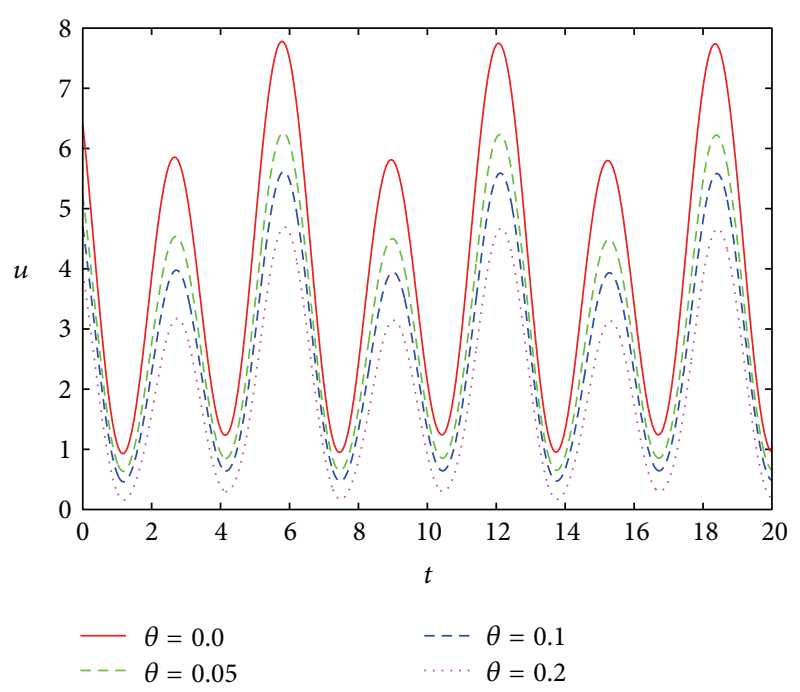

FIGURE 4: Variation of central line velocity with time $t$ for different values of $\theta$, when $n=2, e=0.2, B=0.4, \alpha^{2}=0.05, \epsilon=0.3, \omega=2.0$, $\phi=0.0$, and $\beta=-0.5$.

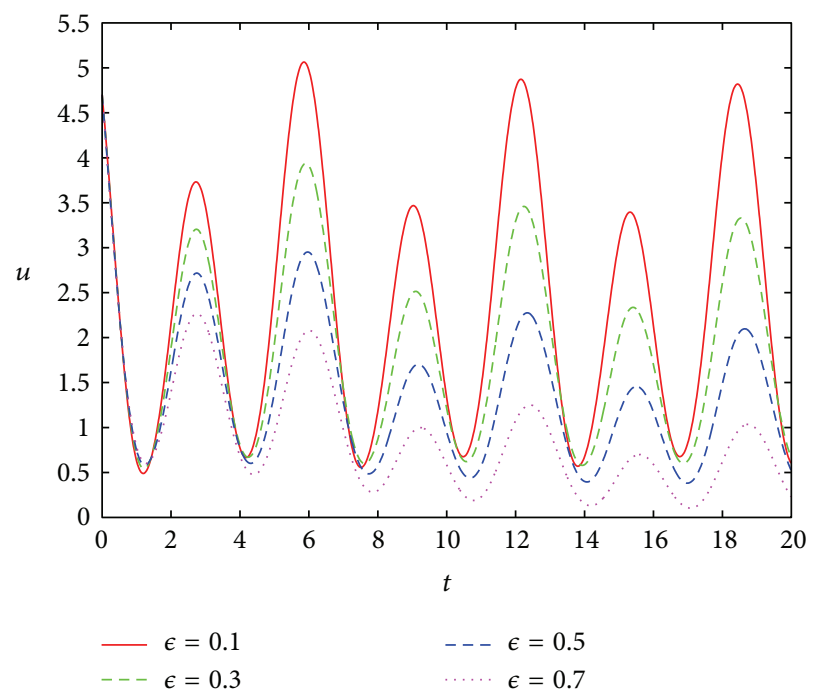

FIGURE 5: Variation of central line velocity with time $t$ for different values of $\epsilon$, when $n=2, e=0.2, B=0.4, \alpha^{2}=0.05, \beta=-0.5$, $\omega=2.0, \phi=0.0$, and $\theta=0.1$.

The distributions of flow rate for different values of velocity slip, yield stress, body acceleration parameter, and pressure gradient parameter have also been computed. The results are plotted and presented graphically through Figures $7-9$. Figure 7 reveals that the volumetric flow rate increases as the values of the velocity slip increases. It can also be noted that in the stenotic region, initially the velocity increases along with the axis of the artery attaining its maximum and then it decreases monotonically. One may note from Figure 8 that for any values of yield stress $\theta$, flow rate oscillates with time. It has been seen from the same figure that for each oscillation, trough/crest is maximum for minimum value of yield stress. Figure 9 gives the variation of volumetric flow 


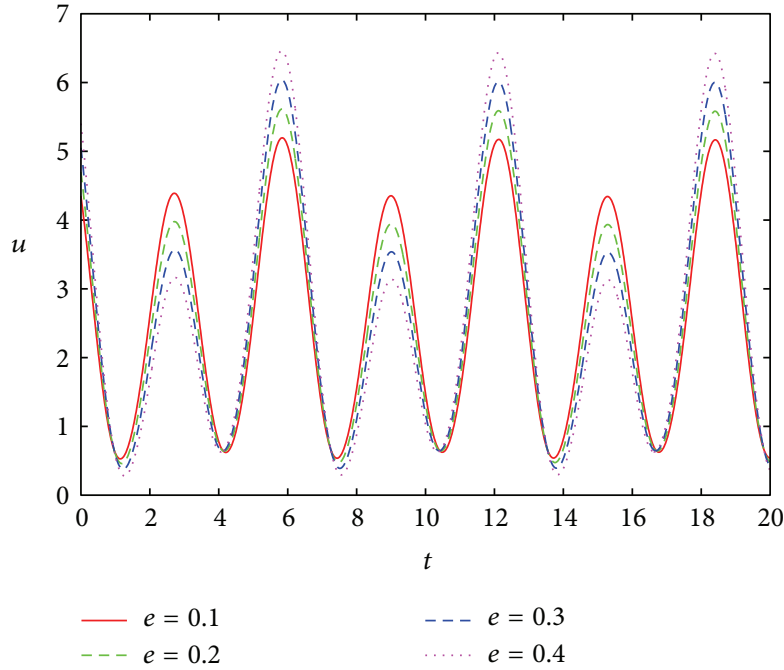

FIGURE 6: Variation of central line axial velocity with time $t$ for different values of $e$, when $n=2, \beta=-0.5, B=0.4, \alpha^{2}=0.05$, $\epsilon=0.3, \omega=2.0, \phi=0.0$, and $\theta=0.1$.

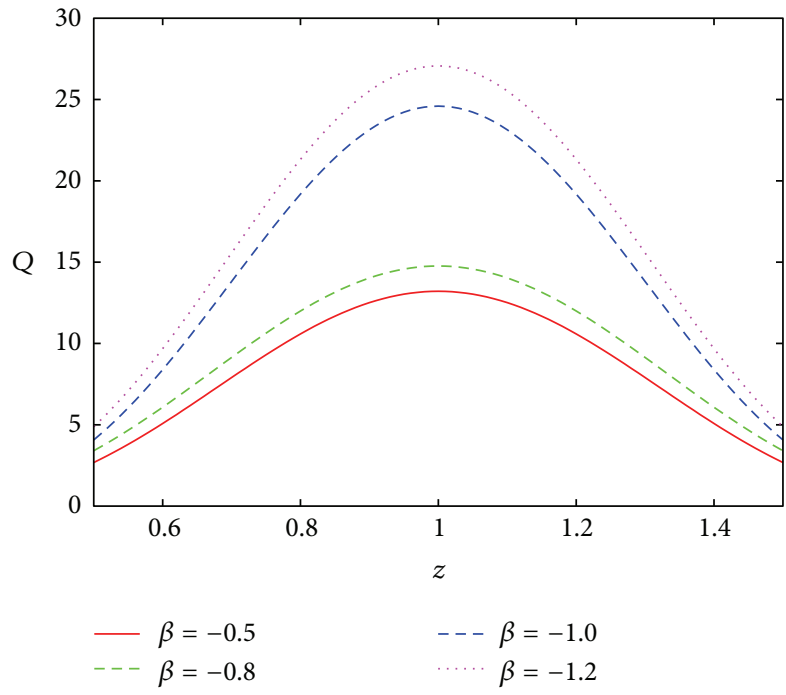

FIGURE 7: Variation of volumetric flow rate for different values of $\beta$ when $n=2, e=0.2, B=0.4, \alpha^{2}=0.05, \epsilon=0.3, \omega=2.0, \phi=$ $0.0, \theta=0.1$, and $t=\pi / 4$.

rate with pressure gradient parameter $e$, for different values of body acceleration parameter $B$. This figure shows that flow rate decreases with pressure gradient parameter $e$, whereas the flow rate increases as the body acceleration parameter increases. Therefore, the pressure gradient parameter $e$ has a linear relation with flow rate $Q$ with decreasing trend.

The computational results for the wall shear stress computed on the basis of the present study are presented in Figures 10-12 for different values of the parameters $\beta, B$, and $\theta$, respectively. Figure 10 shows that for any values of $\beta$, the wall shear stress oscillates with time. Also it has been observed from this figure that the wall shear stress decreases as the velocity-slip increases when $t \in[1.5,3.2],[4.5,6.1]$ and

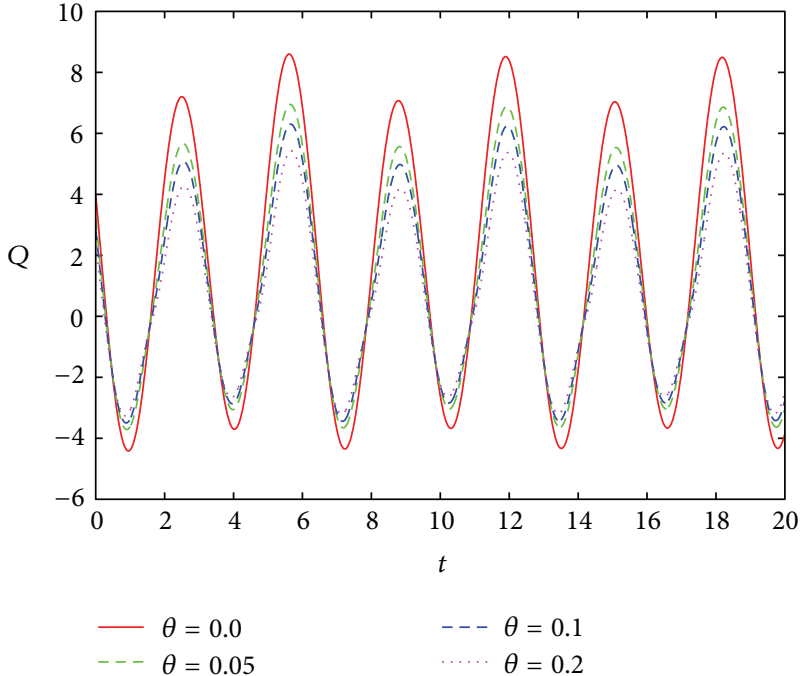

FIGURE 8: Variation of volumetric flow rate for different values of $\theta$ when $n=2, e=0.2, B=0.4, \alpha^{2}=0.05, \epsilon=0.3, \omega=2.0, \phi=0.0$, $\beta=-0.5$, and $t=\pi / 4$.

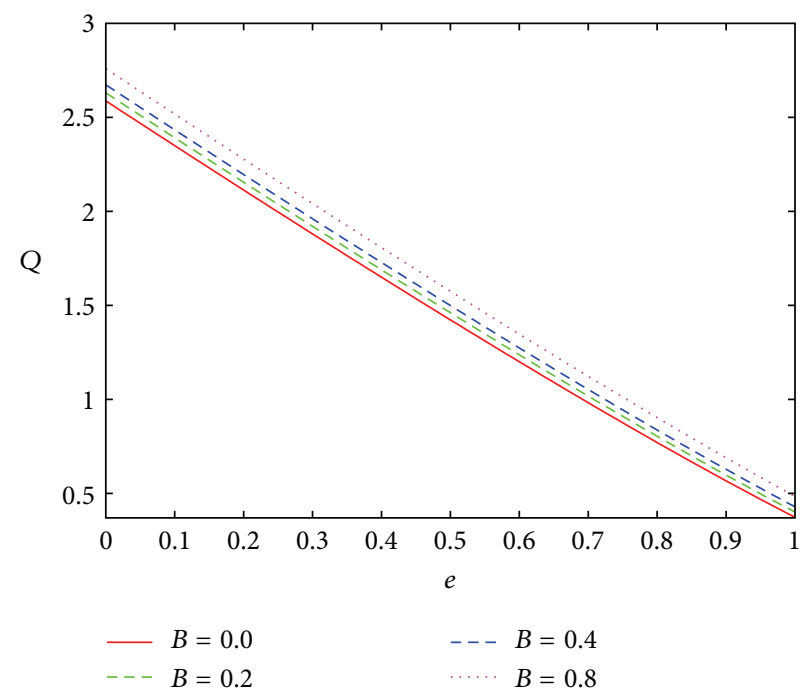

FIGURE 9: Variation of volumetric flow rate with $e$ for different values of $B\left(n=2, \alpha^{2}=0.05, \theta=0.1, \epsilon=0.3, \omega=2.0, \phi=0.0, \beta=-0.5\right.$, and $t=\pi / 4)$.

so on, while the trend is reversed in rest of the time intervals. Figure 11 depicts the distribution of wall shear stress for different values of body acceleration parameter $B$. One may note from this figure that the period of oscillation increases as the body acceleration parameter decreases. Figure 12 represents the wall shear stress distribution in the stenotic region for different values of yield stress. This figure indicates that the wall shear stress decreases as $\theta$ increases. We also observed that the maximum wall shear stress occurs at the throat of the stenosis.

The variation of flow resistance with the height of stenosis is shown in Figure 13 for different values of slip velocity parameter $\beta$. The results presented in this figure reveal 


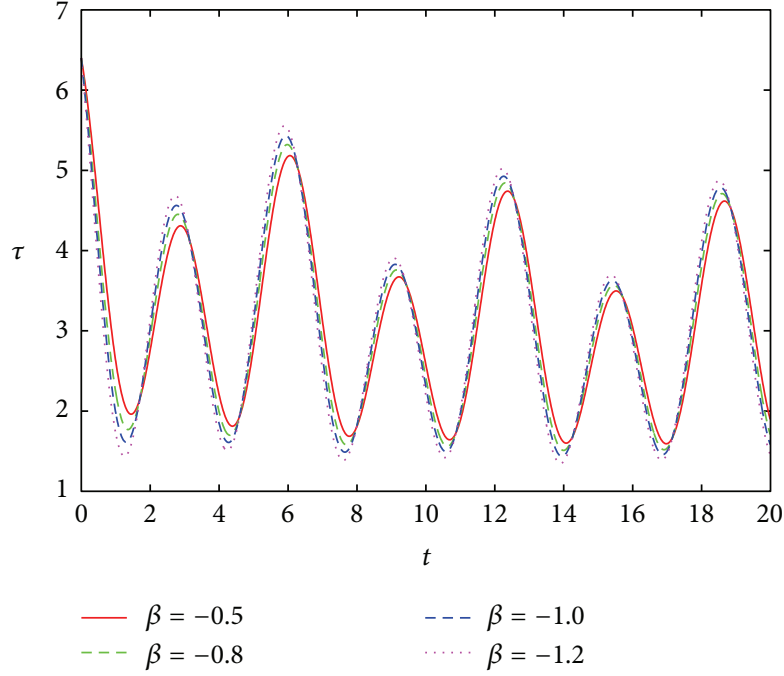

FIGURE 10: Distribution of wall shear stress $(\tau)$ at $z=1.0$ for different values of $\beta$ with $n=2, e=0.2, B=0.4, \alpha^{2}=0.05, \epsilon=0.3, \omega=2.0$, $\phi=0.0, \theta=0.1$, and $t=\pi / 4$.

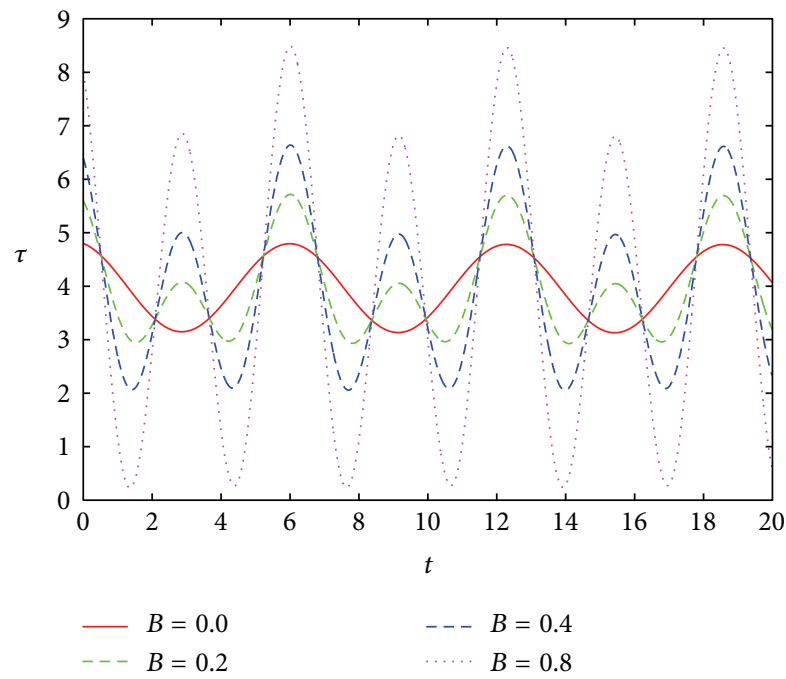

FIGURE 11: Variation of the wall shear stress $(\tau)$ with time at $z=1.5$ for different values of $B$ with $n=2, e=0.2, \beta=-0.5, \alpha^{2}=0.05$, $\epsilon=0.3, \omega=2.0, \phi=0.0$, and $\theta=0.1$.

that the flow resistance increases with the increase in the stenosis height and that as the velocity slip increases, the flow resistance decreases. Figure 14 gives the variation of flow resistance with the maximum height of the stenosis for different values of the body acceleration parameter $B$. This figure shows that the body acceleration has a reducing effect on the flow resistance.

\section{Concluding Remarks}

The present analysis deals with a theoretical investigation of blood flow characteristics through a narrow and timedependent stenosed artery in the presence of body

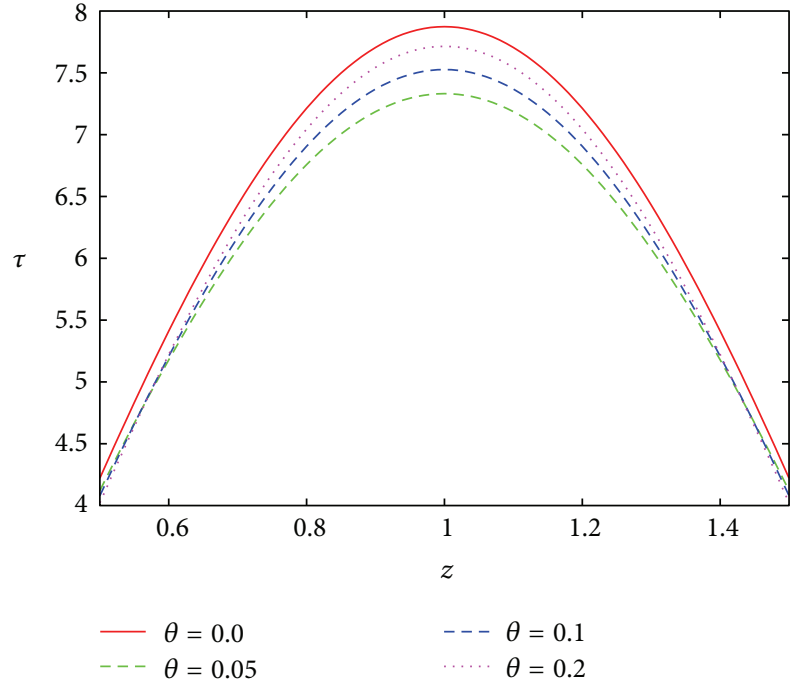

FIGURE 12: Variation of the wall shear stress $(\tau)$ for different values of $\theta\left(n=2, e=0.2, B=0.4, \alpha^{2}=0.05, \epsilon=0.3, \omega=2.0, \phi=0.0\right.$, $\beta=-0.5$, and $t=\pi / 4)$.

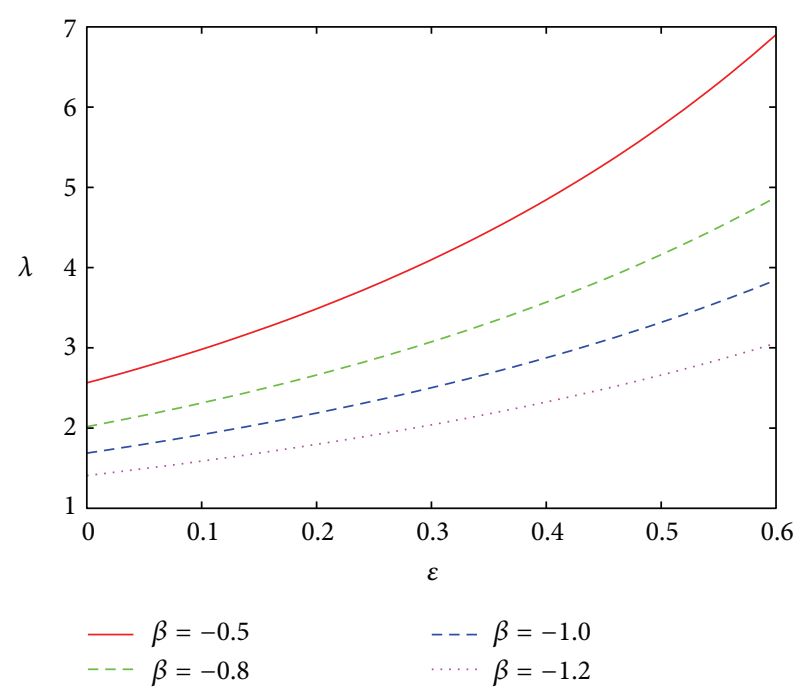

FIGURE 13: Variation of the resistance to the flow with $\epsilon$ for different values of $\beta$ when $n=2, e=0.2, B=0.4, \alpha^{2}=0.05, \omega=2.0, \phi=0.0$, $\theta=0.1$, and $t=\pi / 4$.

acceleration and velocity slip by treating it as a Casson fluid model. Using the appropriate boundary conditions, analytical expressions for the velocity, wall shear stress, and flow resistance have been estimated. The computational results were presented graphically for different values of the parameters involved in the present problem under consideration. Figure 15 shows the comparison of our results with those of computer generated results [32] in order to validate the present mathematical model. For the purpose of comparison, both the studies have been naturally brought to the same platform, by disregarding the slip effects and by considering $T$ being very small for the present study, while for the previous study the inclination angle has been considered to be zero. 


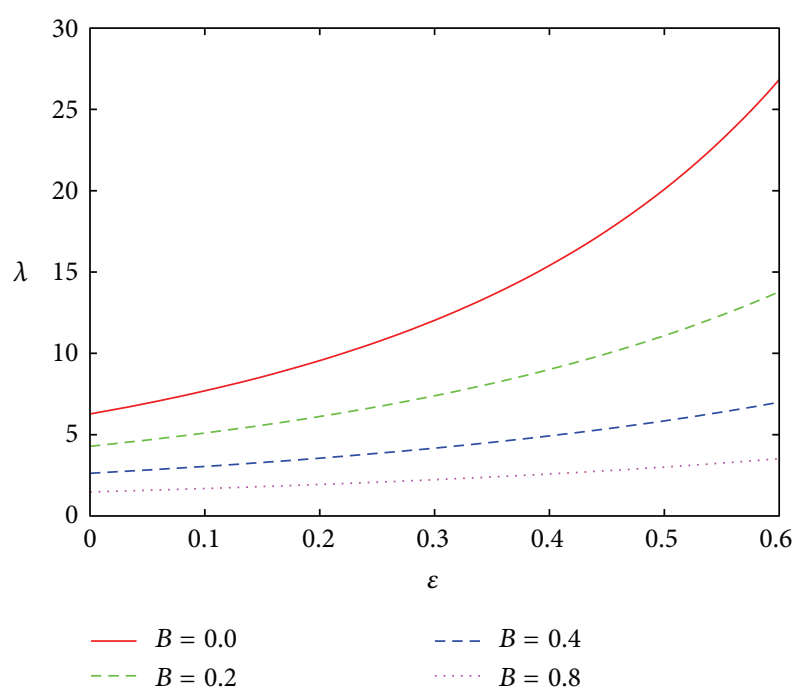

FIGURE 14: Variation of the resistance to the flow with $\epsilon$ for different values of $B$ when $n=2, e=0.2, \beta=-0.5, \alpha^{2}=0.05, \omega=2.0$, $\phi=0.0, \theta=0.1$, and $t=\pi / 4$.

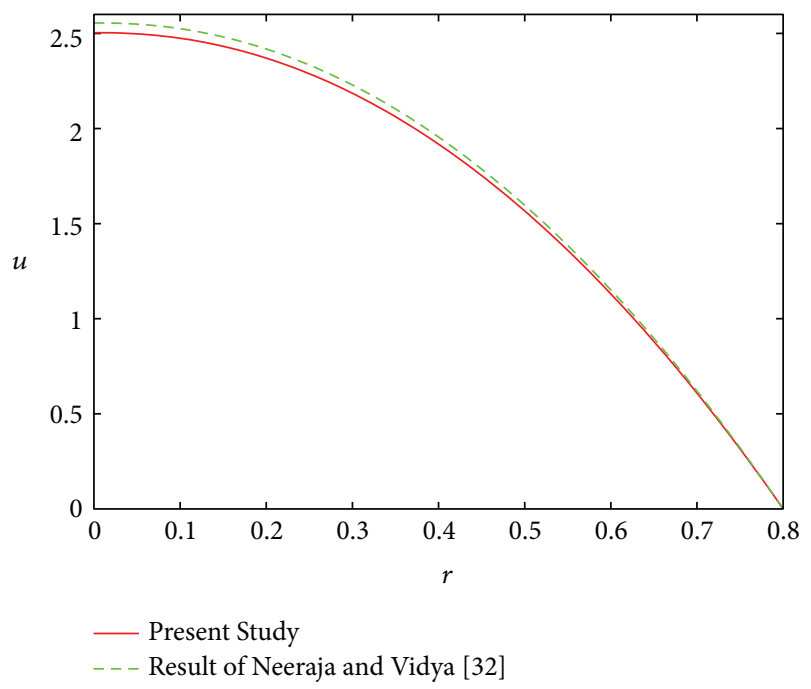

FIGURE 15: Comparison of axial velocity with those of Neeraja and Vidya [32] when $\beta=0.0, T=0.1, \epsilon=0.2, B=1.0, \omega=2.0$, $\alpha^{2}=0.01, \theta=0.01$, and $n=2$.

The present study bears the potential to examine the complex flow behavior of blood under the simultaneous influence of different factors like the size of the stenosis, body acceleration parameter, pressure gradient parameter, and the velocity slip. The observations made on the basis of the present study are quite significant. The investigation shows that the blood velocity increases as body acceleration parameter increases. This fact is harmful for the heart.

Caro et al. [33] made a conjecture that the arterial disease atheroma develops in the regions where the mean wall shear stress is relatively low. Experimental studies also revealed that low velocity regions are prone to the development of atherosclerosis/further deposition.
The main objective in our present study has been to assess the role of velocity slip in blood flow through arteries and to determine those regions where the velocity is low and also the regions where the wall shear stress is low. Thus the study bears the potential to further explore the causes and development of arterial diseases like atherosclerosis and atheroma.

An increase in the size of stenoses causes enhancement of the resistance to blood flow through the arteries in the brain, heart, and other organs of the body. This may lead to stroke, heart attack, and various other cardiovascular diseases.

\section{Nomenclature}

$u^{\prime}: \quad$ Velocity of blood

$p^{\prime}: \quad$ Pressure

$\tau^{\prime}: \quad$ Shear stress

$\tau_{y}: \quad$ Yield stress

$t: \quad$ Time

$d^{\prime}$ : The distance of the onset of stenosis from entrance

$G^{\prime}\left(t^{\prime}\right)$ : Body acceleration

$l_{0}^{\prime}$ : $\quad$ Length of stenosis

$r_{0}^{\prime}$ : $\quad$ Radius of plug

$R_{0}$ : $\quad$ Radius of the pericardial surface of normal portion of the arterial segment

$R^{\prime}$ : $\quad$ Radius of the endothelium of the stenosed portion

$n(\geq 2)$ : Shape parameter of stenosis

$\epsilon^{\prime}: \quad$ Maximum height of the stenosis

$\mu$ : $\quad$ Coefficient of viscosity

$\rho: \quad$ Density of blood

$\beta^{\prime}: \quad$ Slip coefficient

$\omega_{1}^{\prime}$ : $\quad$ Angular frequency

$a_{0}$ : Amplitude of acceleration

$\phi$ : $\quad$ Phase difference

$f_{2}$ : $\quad$ Pulse frequency

$\alpha^{2}$ : Womersley parameter

$e: \quad$ Pressure gradient parameter

$\theta: \quad$ Nondimensional yield stress

$B$ : $\quad$ Body acceleration parameter

$\beta$ : $\quad$ Slip length.

\section{Acknowledgments}

One of the authors (A. Sinha) is grateful to the NBHM, DAE, Mumbai, India, for the financial support of this investigation. This work is also partly supported by DST, New Delhi, Government of India, through project Grant no. SR/FTP/MS042/2011.

\section{References}

[1] C. Clark, "Turbulent velocity measurements in a model of aortic stenosis," Journal of Biomechanics, vol. 9, no. 11, pp. 677-687, 1976.

[2] G. S. Beavers and D. D. Joseph, "Boundary conditions at a naturally permeable wall," Journal of Fluid Mechanics, vol. 30, no. 1, pp. 197-207, 1967. 
[3] L. M. Srivastava, "Flow of couple stress fluid through stenotic blood vessels," Journal of Biomechanics, vol. 18, no. 7, pp. 479485, 1985.

[4] C. Tu, M. Deville, L. Dheur, and L. Vanderschuren, "Finite element simulation of pulse tile flow through arterial stenosis," Journal of Biomechanics, vol. 25, no. 10, pp. 1141-1152, 1992.

[5] S. Chakravarty, "Effects of stenosis on the flow-behaviour of blood in an artery," International Journal of Engineering Science, vol. 25, no. 8, pp. 1003-1016, 1987.

[6] J. C. Misra and G. C. Shit, "Blood flow through arteries in a pathological state: a theoretical study," International Journal of Engineering Science, vol. 44, no. 10, pp. 662-671, 2006.

[7] J. B. Shukla, R. S. Parihar, and S. P. Gupta, "Effects of peripheral layer viscosity on blood flow through the artery with mild stenosis," Bulletin of Mathematical Biology, vol. 42, no. 6, pp. 797-805, 1980.

[8] Q. Long, X. Y. Xu, K. V. Ramnarine, and P. Hoskins, "Numerical investigation of physiologically realistic pulsatile flow through arterial stenosis," Journal of Biomechanics, vol. 34, no. 10, pp. 1229-1242, 2001.

[9] M. Texon, "The hemodynamic concept of atherosclerosis," National Library of Medicine, vol. 36, no. 4, pp. 263-273, 1960.

[10] J. C. Misra, A. Sinha, and G. C. Shit, "Mathematical modeling of blood flow in a porous vessel having double stenoses in the presence of an external magnetic field," International Journal of Biomathematics, vol. 4, no. 2, pp. 207-225, 2011.

[11] J. C. Misra, S. D. Adhikary, and G. G. Shit, "Mathematical analysis of blood flow through an arterial segment with timedependent stenosis," Mathematical Modelling and Analysis, vol. 13, no. 3, pp. 401-412, 2008.

[12] A. Sarkar and G. Jayaraman, "Correction to flow rate-pressure drop relation in coronary angioplasty: steady streaming effect," Journal of Biomechanics, vol. 31, no. 9, pp. 781-791, 1998.

[13] M. El-Shahed, "Pulsatile flow of blood through a stenosed porous medium under periodic body acceleration," Applied Mathematics and Computation, vol. 138, no. 2-3, pp. 479-488, 2003.

[14] E. F. Elshehawey, E. M. E. Elbarbary, N. A. S. Afifi, and M. El-Shahed, "Pulsatile flow of blood through a porous medium under periodic body acceleration," International Journal of Theoretical Physics, vol. 39, no. 1, pp. 183-188, 2000.

[15] P. Nagarani and G. Sarojamma, "Effect of body acceleration on pulsatile flow of casson fluid through a mild stenosed artery," Korea Australia Rheology Journal, vol. 20, no. 4, pp. 189-196, 2008.

[16] S. U. Siddiqui, N. K. Verma, S. Mishra, and R. S. Gupta, "Mathematical modelling of pulsatile flow of Casson's fluid in arterial stenosis," Applied Mathematics and Computation, vol. 210, no. 1, pp. 1-10, 2009.

[17] V. K. Sud and G. S. Sekhon, "Arterial flow under periodic body acceleration," Bulletin of Mathematical Biology, vol. 47, no. 1, pp. 35-52, 1985.

[18] V. K. Sud and G. S. Sekhon, "Analysis of blood flow through a model of the human arterial system under periodic body acceleration," Journal of Biomechanics, vol. 19, no. 11, pp. 929941, 1986.

[19] P. Chaturani and V. Palanisamy, "Pulsatile flow of power-law fluid model for blood flow under periodic body acceleration," Biorheology, vol. 27, no. 5, pp. 747-758, 1990.

[20] S. N. Majhi and V. R. Nair, "Pulsatile flow of third grade fluids under body acceleration-modelling blood flow," International Journal of Engineering Science, vol. 32, no. 5, pp. 839-846, 1994.
[21] G. C. Shit and M. Roy, "Pulsatile flow and heat transfer of a magneto-micropolar fluid through a stenosed artery under the influence of body acceleration," Journal of Mechanics in Medicine and Biology, vol. 11, no. 3, pp. 643-661, 2011.

[22] J. C. Misra, M. K. Patra, and S. C. Misra, "A non-Newtonian fluid model for blood flow through arteries under stenotic conditionse," Journal of Biomechanics, vol. 26, no. 9, pp. 11291141, 1993.

[23] J. C. Misra and G. C. Shit, "Role of slip velocity in blood flow through stenosed arteries: a non-Newtonian model," Journal of Mechanics in Medicine and Biology, vol. 7, no. 3, pp. 337-353, 2007.

[24] P. Brunn, “The velocity slip of polar fluids," Rheologica Acta, vol. 14, no. 12, pp. 1039-1054, 1975.

[25] Y. Nubar, "Blood flow, slip, and viscometry," Biophysical Journal, vol. 11, no. 3, pp. 252-264, 1971.

[26] R. Ponalagusamy, "Blood flow through an artery with mild stenosis: a two-layered model, different shapes of stenoses and slip velocity at the wall," Journal of Applied Sciences, vol. 7, no. 7, pp. 1071-1077, 2007.

[27] D. Biswas and U. S. Chakraborty, "Pulsatile flow of blood in a constricted artery with body acceleration," Applications and Applied Mathematics, vol. 4, pp. 329-342, 2009.

[28] D. Biswas and U. S. Chakraborty, "Pulsatile blood flow through a catheterized artery with an axially nonsymmetrical stenosis," Applied Mathematical Sciences, vol. 4, no. 57-60, pp. 2865-2880, 2010.

[29] A. H. Shapiro, M. Y. Jaffrin, and S. L. Weinberg, "Peristaltic pumping with long wavelength at low Reynolds number," Journal of Fluid Mechanics, vol. 37, no. 4, pp. 799-825, 1969.

[30] S. Takabatake, K. Ayukawa, and A. Mori, "Peristaltic pumping in circular cylindrical tubes: a numerical study of fluid transport and its efficiency," Journal of Fluid Mechanics, vol. 193, pp. 267283, 1988.

[31] P. Chaturani and R. P. Samy, "Pulsatile flow of Casson's fluid through stenosed arteries with applications to blood flow," Biorheology, vol. 23, no. 5, pp. 499-511, 1986.

[32] G. Neeraja and K. Vidya, "Effect of body acceleration on pulsatile flow of Herschel-Bulkley fluid through an inclined mild stenosed artery," International Journal of Engineering Research \& Technology, vol. 1, pp. 1-10, 2012.

[33] C. G. Caro, J. M. Fitz-Gerald, and R. C. Schroter, "Atheroma and arterial wall shear stress observation, correlation and proposal of a shear dependent mass transfer mechanism for atherogenesis," Proceedings of the Royal Society of London B, vol. 177, no. 46, pp. 109-159, 1971. 

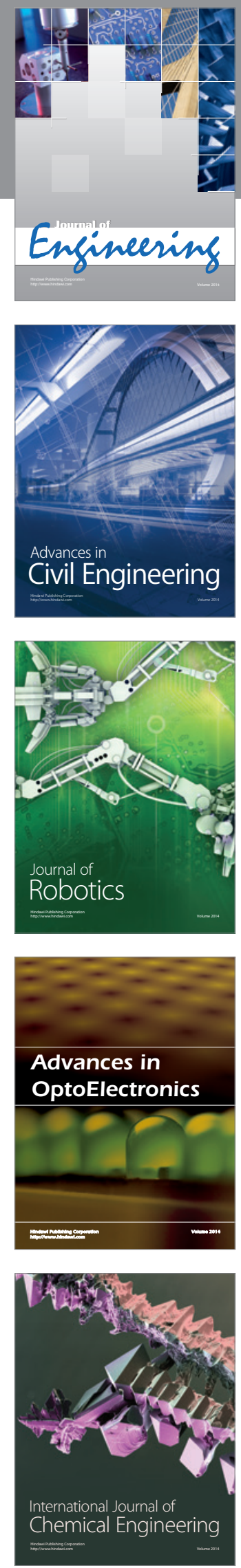

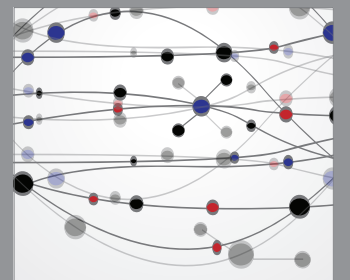

The Scientific World Journal
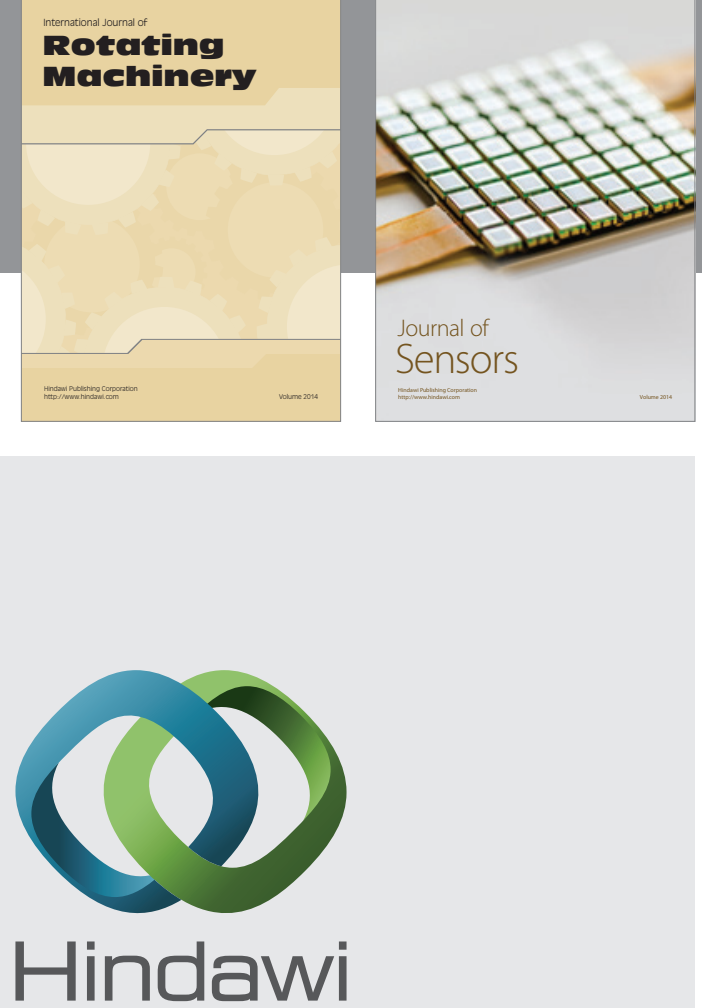

Submit your manuscripts at http://www.hindawi.com
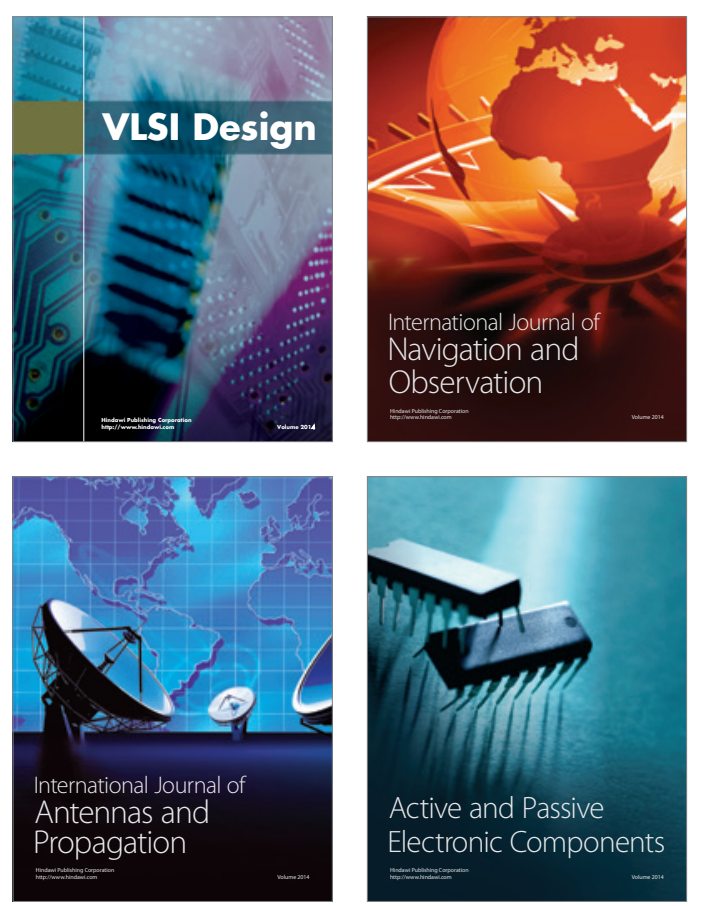
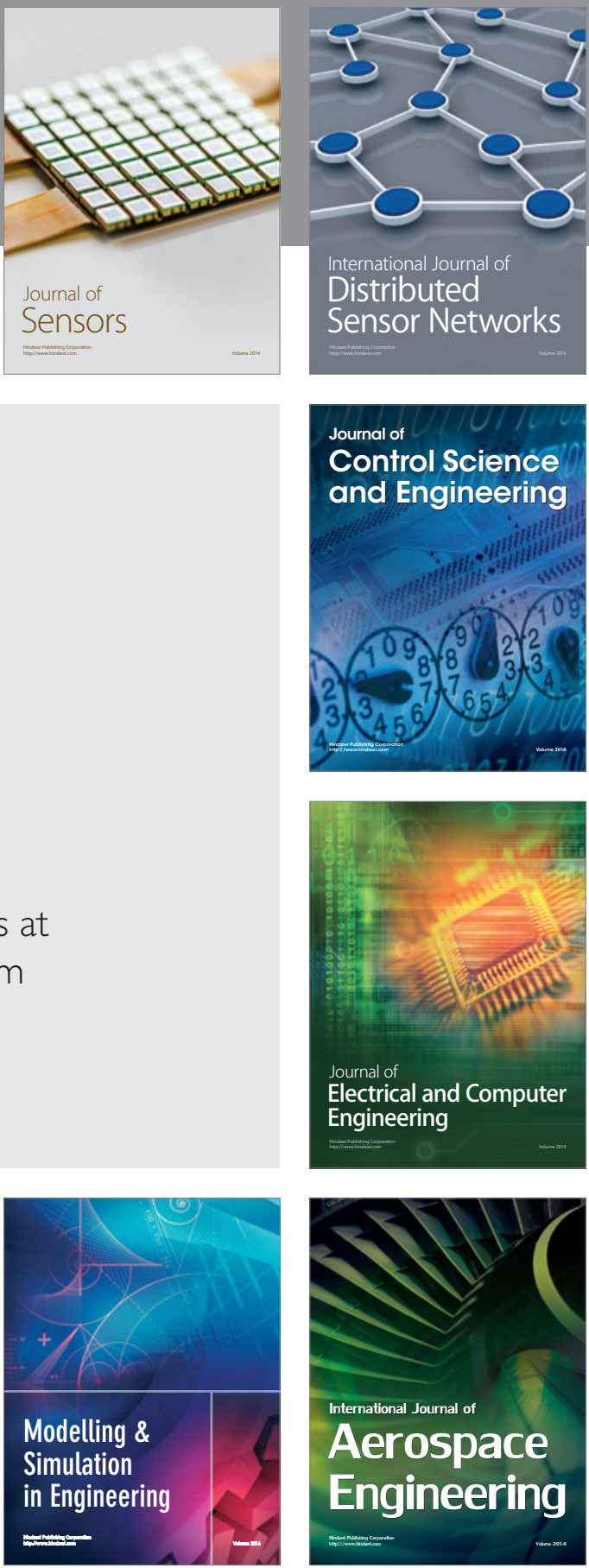

Journal of

Control Science

and Engineering
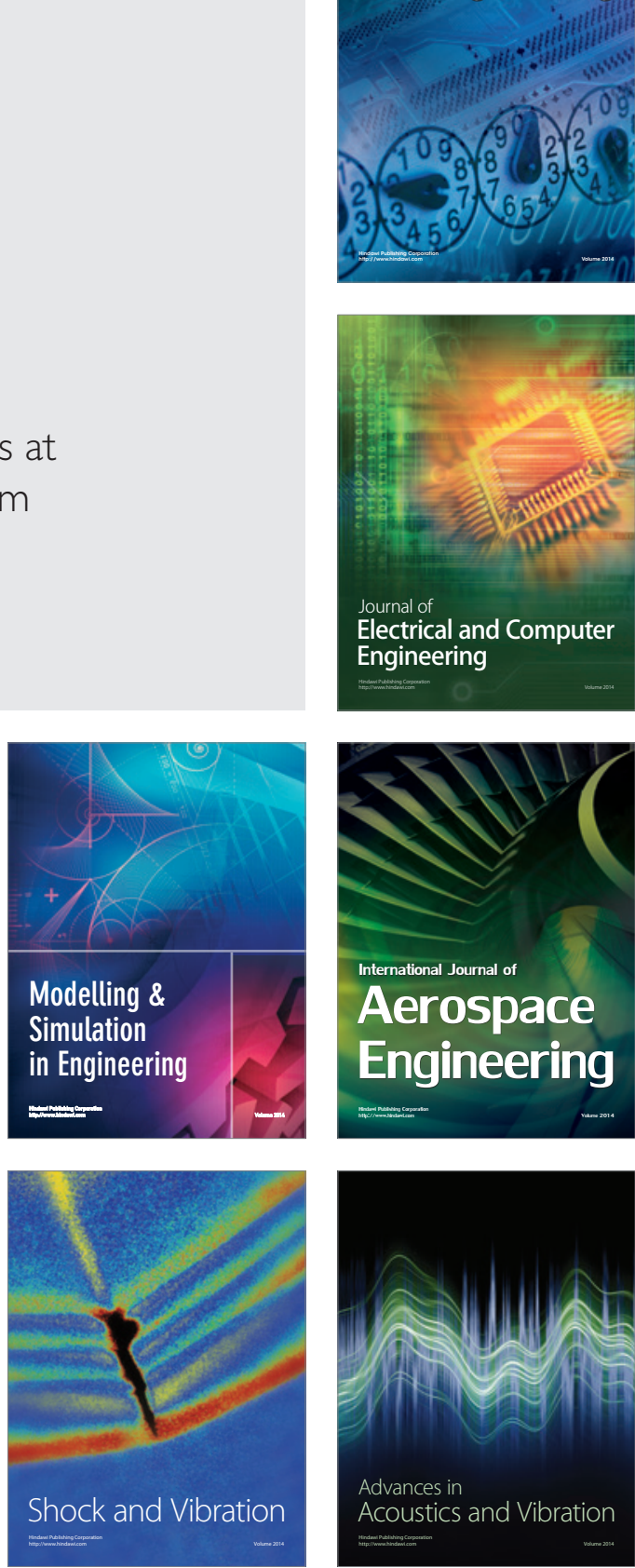